\title{
Downlink Space-Time Spreading Using Interference Rejection Codes
}

\author{
Hua Wei, Lie-Liang Yang, Senior Member, IEEE, and Lajos Hanzo, Fellow, IEEE
}

\begin{abstract}
In this paper, the authors will investigate the performance of a loosely synchronized (LS) code-based space-time spreading (STS) scheme in comparison to that of classic Walsh code and pseudonoise code-based STS when communicating over dispersive Nakagami- $m$ multipath channels. Closed-form formulas are derived for characterizing the bit-error-rate performance as a function of the number of resolvable paths $L$ and the number of users $K$. Our numerical results suggest that the employment of LS code-based STS scheme is beneficial in a low-user-load and low-dispersion channel scenario, where a near-single-user performance can be achieved without a multiuser detector.
\end{abstract}

Index Terms-Code-division multiple access (CDMA), Gaussian approximation, interference-free window (IFW), large area synchronized (LAS) codes, loosely synchronized (LS) codes, Nakagami- $m$ fading.

\section{INTRODUCTION}

$\mathbf{T}$ HE underlying philosophy of space-time spreading (STS) is reminiscent of the operating principles of space-time coding (STC) [1], where multiple replicas of the same symbol are mapped to multiple transmit antennas for the sake of achieving a transmit diversity gain. In the context of STS, the information to be transmitted may be mapped to multiple transmitter antennas with the aid of the STS codes proposed in [2], which were graphically illustrated in [3]. In simple terms, the STS codes are used for spatially spreading the information to multiple transmit antennas, again, for the sake of achieving spatial diversity.

When we consider an STS-assisted direct-sequence codedivision multiple-access (DS-CDMA) scheme communicating over a nondispersive channel, the employment of orthogonal spreading codes such as Walsh codes and orthogonal Gold codes [3] is ideal for the nondispersive synchronous downlink (DL) channel since the channel will not destroy the orthogonality of the codes when we invoke a matched filter-based rake receiver at the receiver side. However, classic orthogonal codes, e.g., Walsh codes, will lose their orthogonality when communicating over a dispersive multipath channel. More specifically, when the rake receiver coherently combines the different paths'

Manuscript received May 8, 2005; revised September 4, 2005 and January 15, 2006. This work was supported in part by the Virtual Centre of Excellence in Mobile and Personal Communications (Mobile VCE; http://www. mobilevce.com), the Engineering and Physical Sciences Research Council, and the EU under the auspices of the NEWCOM and PHOENIX projects. This paper has formed part of the Wireless Enablers Work Area of the Core 2 Research Programme of the Mobile VCE. The review of this paper was coordinated by Prof. J. H. Cho.

The authors are with the School of Electronics and Computer Science, University of Southampton, Southampton SO17 1BJ, U.K. (e-mail: 1h@ecs. soton.ac.uk).

Digital Object Identifier 10.1109/TVT.2006.878607 energy, it will inevitably combine both the multiple access interference (MAI) and the multipath interference (MPI) in case of communicating over a dispersive multipath channel. To circumvent this problem, the family of loosely synchronized (LS) codes [4], [5] has been proposed, which exhibits a socalled interference-free window (IFW). More explicitly, these codes are capable of suppressing both the MAI and MPI, provided that these interfering components arrive within the IFW. Hence, when the dispersive channel's delay spread does not exceed the width of the IFW, we can combine all the paths' energy without imposing any MAI and MPI interference, and hence, interference-free CDMA communication becomes possible without the employment of high-complexity multiuser detection. Furthermore, we will demonstrate that even when the channel's delay spread does exceed the width of the IFW, the proposed LS code-based STS scheme is capable of outperforming the conventional STS scheme. However, the disadvantage of the LS code-based STS scheme advocated is that the number of available LS codes is limited when aiming for a specific spreading gain $G$. More explicitly, the number of supported users and the width of the IFW $\iota$ must satisfy $K(\iota+1) \leq G$ [4]. To expound a little further, we can achieve a high IFW width and suppress the interference more effectively when the number of users supported in the channel is relatively low because the number of codes exhibiting a high IFW is low. By contrast, as the number of users increases, the IFW width tends to zero since all the codes having a wide IFW have been activated, and hence, the LS code-based STS scheme becomes incapable of suppressing the MAI and MPI.

Since at the time of writing no in-depth peer-reviewed IEEE journal paper study exists on the topic of LS code-aided STS CDMA/multicarrier (MC) CDMA, the novelty of this paper is that we investigate the performance of an LS code-based STS scheme in comparison to the benchmarker STS scheme of [2] when communicating over dispersive Nakagami- $m$ multipath channels. Since LS codes were described in [4] and [5], whereas the philosophy of STS was detailed in [2] and [3], here we refrain from their detailed description.

This paper is organized follows: Section II describes the system model used, whereas Section III illustrates the detection of STS signals. Section IV characterizes the achievable biterror-rate (BER) performance, whereas Section V discusses our numerical results. Finally, Section VI offers our conclusions.

\section{A. LS Codes}

LS codes [4] exploit the properties of the so-called orthogonal complementary sets [4], [6]. To expound further, let us 


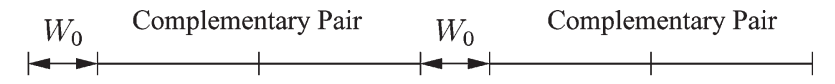

(a)

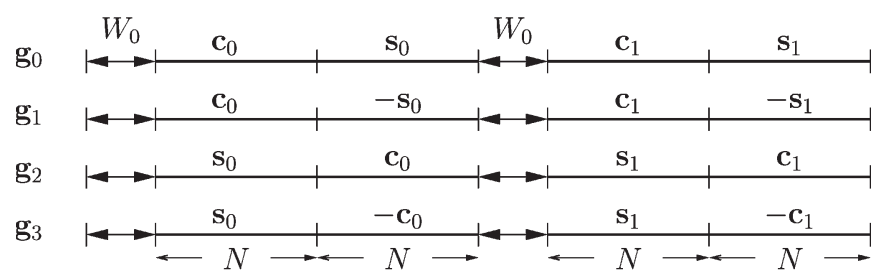

(b)

Fig. 1. Generating the $\operatorname{LS}\left(N, P, W_{0}\right)$ code using the $(P \times P)=(4 \times 4)$ WH matrix components $(1,1,1,1)$ and $(1,-1,1,-1)$. (a) LS code structure. (b) Generating four LS codes.

introduce the notation of $\operatorname{LS}\left(N, P, W_{0}\right)$ for denoting the family of LS codes generated by applying a $(P \times P)$-dimensional Walsh-Hadamard (WH) matrix to an orthogonal complementary code set of length $N$, as it is exemplified in the context of Fig. 1. More specifically, we generate a complementary code pair by inserting $W_{0}$ number of zeros both in the center and at the beginning of the complementary pair, as shown in Fig. 1(a), using the procedure described in [4]. As mentioned above, the polarity of the codes $c_{0}$ and $s_{0}$ shown in Fig. 1(b) during the constitution of the LS codes is determined by the polarity of the components of a WH matrix, namely, by $(1,1,1,1)$ and $(1,-1,1,-1)$. Then, the total length of the $\operatorname{LS}\left(N, P, W_{0}\right)$ code is given by $L_{S}=N P+2 W_{0}$, and later, we will demonstrate that the total number of codes available is given by $4 P$. The number of these codes having an IFW of $W_{0}$ chips is $P$, which limits the number of users that can be supported without imposing multiuser interference. Hence, the number of codes having as long an IFW as possible has to be maximized for a given code length of $L_{S}=N P+2 W_{0}$.

Since the construction method of binary LS codes was described in [4], here, we refrain from providing an in-depth discourse, and we will focus our attention on the employment of orthogonal complementary sets [7], [8] for the generation of LS codes.

For a given complementary code pair $\left\{\mathbf{c}_{0}, \mathbf{s}_{0}\right\}$ of length $N$, one of the corresponding so-called mate pairs can be written as $\left\{\mathbf{c}_{1}, \mathbf{s}_{1}\right\}$, where we have

$$
\begin{aligned}
& \mathbf{c}_{1}=\tilde{\mathbf{s}}_{0}^{*} \\
& \mathbf{s}_{1}=-\tilde{\mathbf{c}}_{0}^{*}
\end{aligned}
$$

where the superscript $*$ represents conjugation. $\tilde{\mathbf{s}}_{0}$ denotes the reverse-ordered sequence, whereas $-\mathbf{s}_{0}$ is the negated version of $\mathbf{s}_{0}$. Note that in (1) and (2), additional complex conjugation of the polyphase complementary sequences $\left\{\mathbf{c}_{0}, \mathbf{s}_{0}\right\}$ is required for deriving the corresponding mate pair $\left\{\mathbf{c}_{1}, \mathbf{s}_{1}\right\}$ in comparison to binary complementary sequences [4]. Having obtained a complementary pair and its corresponding mate pair, we may employ the construction method of [4] for generating a whole family of LS codes. The LS codes generated exhibit an IFW of length $W_{0}$. Hence, we may adopt the choice of $W_{0}=N-1$

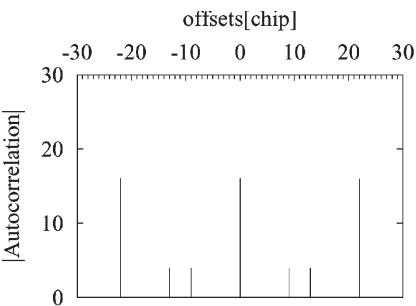

(a)

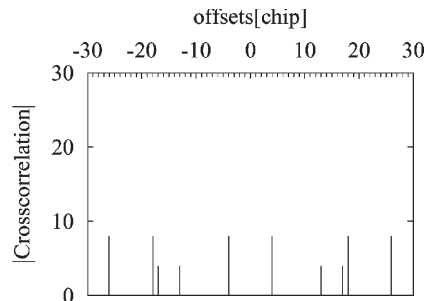

(b)
Fig. 2. Correlation magnitudes of the $\operatorname{LS}(4,4,3)$ codes. (a) All four codes exhibit the same autocorrelation magnitude. (b) Cross-correlation magnitudes of $\mathrm{g}_{0}$ and $\mathbf{g}_{2}$.

to minimize the total length of the LS codes generated while providing as long an IFW as possible.

For example, the $\operatorname{LS}\left(N, P, W_{0}\right)=\mathrm{LS}(4,4,3)$ codes can be generated based on the following complementary pair [7]:

$$
\begin{aligned}
& \mathbf{c}_{0}=+++- \\
& \mathbf{s}_{0}=++-+.
\end{aligned}
$$

Upon substituting (1) and (2) into both (3) and (4), the corresponding mate pair can be obtained as follows:

$$
\begin{aligned}
& \mathbf{c}_{1}=\tilde{\mathbf{s}}_{0}^{*}=+-++ \\
& \mathbf{s}_{1}=-\tilde{\mathbf{c}}_{0}^{*}=+---.
\end{aligned}
$$

The first set of four LS codes can be generated using the first two rows of a $(P \times P)=(4 \times 4)$-dimensional WH matrix, namely, using $\mathbf{w}_{0}=(+1,+1,+1,+1)$ and $\mathbf{w}_{1}=(+1$, $-1,+1,-1)$, as shown in Fig. 1(b). Another set of four LS codes can be obtained by exchanging the subscripts 0 and 1 . Finally, eight additional LS codes can be generated by applying the same principle but with the aid of the last two rows of the $(4 \times 4)$-dimensional WH matrix, namely, using $\mathbf{w}_{2}=(+1,+1,-1,-1)$ and $\mathbf{w}_{3}=(+1,-1,-1,+1)$. Hence, the total number of available codes in the family of $\mathrm{LS}\left(N, P, W_{0}\right)$ is given by $4 P$. More explicitly, there are four sets of $P$ number of LS codes. Each set has four LS codes, and the LS codes in the same set exhibit an IFW length of $[-\iota,+\iota]$, where we have $\iota=\min \left\{W_{0}, N-1\right\}$. The aperiodic autocorrelation and cross-correlation function $\rho_{k k}(\tau), \rho_{j k}(\tau)$ of the codes belonging to the same set will be zero, provided that we have $\tau \leq \iota T_{c}$. Furthermore, the LS codes belonging to the four different sets are still orthogonal to each other at zero timing offset, namely, in a perfectly synchronous environment. However, the LS codes belonging to the four different sets will lose their orthogonality when they have a nonzero code offset. All four different codes in the same set of the $\operatorname{LS}(4,4$, 3 ) code family exhibited the same autocorrelation magnitudes, namely that shown in Fig. 2(a). It can be observed in Fig. 2(a) that the off-peak autocorrelation $R_{p}[\tau]$ becomes zero for $|\tau| \leq$ $W_{0}=3$. The cross-correlation magnitudes $\left|R_{j, k}(\tau)\right|$ depicted in Fig. 2(b) are also zero for $|\tau| \leq W_{0}=3$. Based on the observations made with regard to the aperiodic correlations, we 


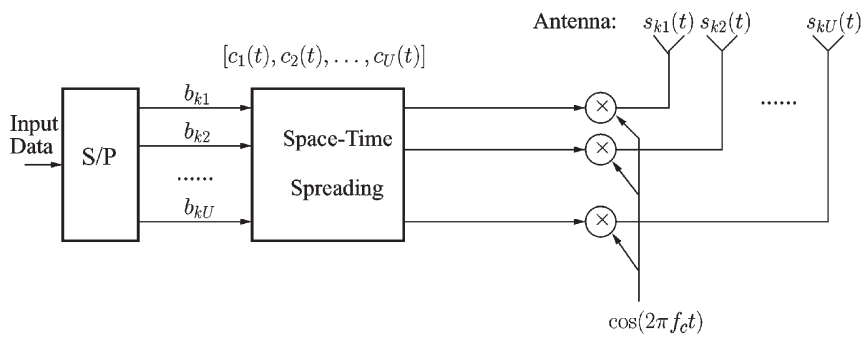

(a)

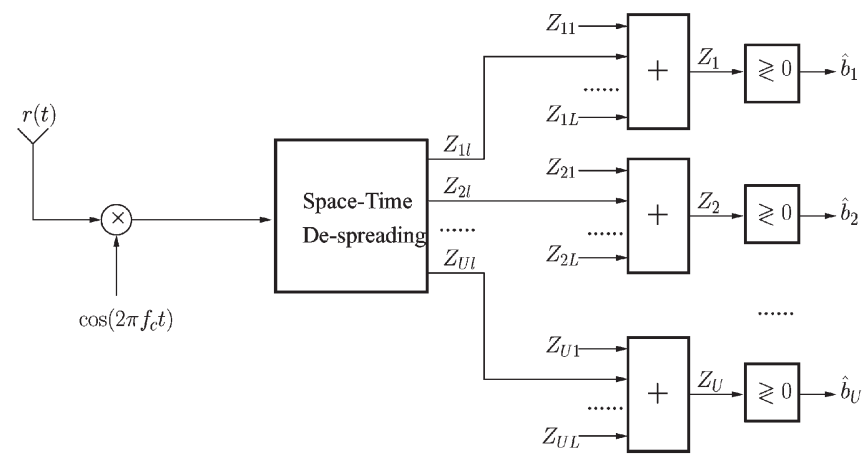

(b)

Fig. 3. Transmitter and receiver block diagram of the W-CDMA system using STS. (a) Transmitter. (b) Receiver.

may conclude that the $\operatorname{LS}(4,4,3)$ codes exhibit an IFW of \pm 3 chip durations.

\section{STS USING INTERFERENCE REJECTION CODES}

\section{A. Transmitted Signal}

As shown in Fig. 3, the system considered in this paper consists of $U$ antennas located at the transmitter side. The binary input data stream having a bit duration of $T_{b}$ is serialto-parallel ( $\mathrm{S} / \mathrm{P})$ converted to $U$ parallel substreams. The new bit duration of each reduced-rate parallel substream, which we refer to as the symbol duration, becomes $T_{s}=U T_{b}$. After $\mathrm{S} / \mathrm{P}$ conversion, the $U$ number of parallel bits that have a $U$-fold higher bit duration are direct-sequence spread using the STS schemes proposed in [2] with the aid of $U$ number of orthogonal spreading sequences, e.g., Walsh codes, having a period of $U G$, where $G=T_{b} / T_{c}$ represents the number of chips per bit, and $T_{c}$ is the chip duration of the orthogonal spreading sequences.

As described above, based on the recommendations of [2], we have assumed that the number of parallel data substreams, the number of orthogonal spreading sequences used by the STS block of Fig. 3, and the number of transmission antennas are the same: namely $U$. This specific STS scheme constitutes a subclass of the generic family of STS schemes, where the number of parallel data substreams, the number of orthogonal spreading sequences required by the STS block, and the number of transmission antennas may take different values. However, the study conducted in [2] has shown that the number of orthogonal spreading sequences required by STS is usually higher than the number of parallel substreams. The STS scheme has an equal number of parallel substreams, orthogonal STSrelated spreading sequences, and transmission antennas, and constitutes an attractive scheme since this STS scheme is capa- ble of providing maximal transmit diversity without requiring extra STS codes [2]. Note that for the specific values of $U=$ 2,4 , and 8 , the aforementioned attractive STS schemes have been specified in [2]. In this section, we only investigate these attractive STS schemes.

Based on the philosophy of STS as discussed in [2] and referring to Fig. 3(a), the transmitted signal of the $k$ th user can be written as

$$
\mathbf{s}_{k}(t)=\sqrt{\frac{2 P}{U^{2}}} \mathbf{c}(t) \mathbf{B}_{U}(t) \times \cos \left(2 \pi f_{c} t\right)
$$

where $P$ represents each user's transmitted power, which is constant for all users, $\mathbf{s}_{k}(t)=\left[s_{k 1}(t) s_{k 2}(t) \cdots s_{k U}(t)\right]$ represents the transmitted signal vector of the $U$ transmission antennas, while $\mathbf{c}(t)$ and $f_{c}$ represent the DS scrambling-based spreading waveform and the carrier frequency, respectively. In (7), the vector $\mathbf{c}(t)=\left[c_{1}(t) c_{2}(t) \cdots c_{U}(t)\right]$ is constituted by the $U$ number of spreading waveforms assigned for the STS block, where $c_{i}(t)=\sum_{j=0}^{U G} c_{i j} P_{T_{c}}\left(t-j T_{c}\right), i=1,2, \ldots, U$ denotes the individual components of the STS-based spread signals, and $\left\{c_{i j}\right\}$ represents a spreading sequence of period $U G$ for each index $i$, while $P_{T_{c}}(t)$ is the rectangular chip waveform spanning the chip interval $\left[0, T_{c}\right]$. In this paper, we will consider two different STS schemes. The benchmarker arrangement is the traditional STS scheme of [2], which is employed, e.g., in wideband CDMA (W-CDMA). In this scheme, $c_{i}(t)$ can be expressed as $c_{i}(t)=w_{i}(t) \otimes \mathrm{PN}(t)=\sum_{j=0}^{U G}\left(w_{i j} \otimes\right.$ $\left.p_{i j}\right) P_{T_{c}}\left(t-j T_{c}\right)$, where $w_{i}(t)=\sum_{j=0}^{U G} w_{i j} P_{T_{c}}\left(t-j T_{c}\right)$ denotes the unique user-specific Walsh spreading sequence used by the STS scheme of Fig. 3, whereas $\mathrm{PN}(t)=$ $\sum_{j=0}^{U G} p_{i j} P_{T_{c}}\left(t-j T_{c}\right)$ is the random cell-specific pseduonoise scrambling sequence. Hence, we have $c_{i j}=w_{i j} \otimes p_{i j}$, and $c_{i j}$ may also be modeled by a random spreading sequence. The employment of the PN scrambling sequence in combination with the Walsh code allows the system to reuse the user-specific Walsh codes in adjacent cells and reduce the MAI. In contrast to the benchmarker, in our proposed scheme, STS is carried out using the family of interference rejection LS codes. In this scheme, the spreading signature waveform $c_{i}(t)$ can be expressed as $c_{i}(t)=\mathrm{LS}_{i}(t)=\sum_{j=0}^{U G} \mathrm{LS}_{i j} P_{T_{c}}\left(t-j T_{c}\right)$, where $\mathrm{LS}_{i}(t)$ denotes the $i$ th LS spreading signature waveform. Moreover, we do not impose PN code-based scrambling on the LS STS codes because this would destroy their IFW. Hence, for the sake of preserving the IFW of the spreading codes, the STS scheme using LS codes refrains from invoking the conventional scheme's scrambling operation. It is worth noting that the omission of the PN code-based spreading does not constitute a problem in conjunction with LS codes since they exhibit an IFW, and hence, they are more immune to both MAI and MPI than the Walsh codes. It has to be noted, however, that owing to the absence of the PN scrambling code, the amount of intercell interference is expected to be higher especially because the adjacent-cell interference is likely to arrive outside the IFW, where the cross correlation of the LS codes is higher than that of the cell-specific PN scrambling codes. Still considering (7), $\mathbf{B}_{U}(t)$ represents the $(U \times U)$-dimensional transmitted data matrix created by mapping $U$ input data bits to the $U$ parallel 
substreams according to the specific design rules of [2], so that the maximum possible transmit diversity is achieved while using relatively low-complexity signal detection algorithms.

\section{B. Channel Model}

The $U$ number of parallel signals $\mathbf{s}_{k}(t)=\left[s_{k 1}(t) s_{k 2}(t)\right.$ $\left.\cdots s_{k U}(t)\right]$ are transmitted by the $U$ number of antennas over frequency-selective fading channels, where each parallel signal experiences independent frequency-selective Nakagami$m$ fading. The complex lowpass equivalent representation of the impulse response experienced by the $u$ th parallel signal of all users is given by [9]

$$
h^{u}(t)=\sum_{l=1}^{L} h_{l}^{u} \delta\left(t-\tau_{l}\right) \exp \left(j \phi_{l}^{u}\right)
$$

where $h_{l}^{u}, \tau_{l}$, and $\psi_{l}^{u}$ represent the attenuation, delay, and phase shift of the lth multipath component of the channel, respectively. Without loss of generality, we assume that we have $\tau_{l}=(l-1) T_{c}$, while $L$ is the total number of resolvable multipath components, and $\delta(t)$ is the Kronecker delta function. We assume that the phases $\left\{\psi_{l}^{u}\right\}$ in (8) are independent identically distributed (i.i.d.) random variables uniformly distributed in the interval $[0,2 \pi)$, while the $L$ multipath attenuations $\left\{h_{l}^{u}\right\}$ in (8) are independent Nakagami random variables having a probability density function (pdf) of [10]-[12]

$$
\begin{aligned}
p\left(h_{l}^{u}\right) & =M\left(h_{l}^{u}, m_{l}^{(u)}, \Omega_{l}^{u}\right) \\
M(R, m, \Omega) & =\frac{2 m^{m} R^{2 m-1}}{\Gamma(m) \Omega^{m}} e^{(-m / \Omega) R^{2}}
\end{aligned}
$$

where $\Gamma(\cdot)$ is the gamma function [9], and $m_{k l}^{(u)}$ is the Nakagami- $m$ fading parameter, which characterizes the severity of the fading over the $l$ th resolvable path [13] between the $u$ th transmission antenna and user $k$. Specifically, $m_{l}^{(u)}=1$ represents Rayleigh fading, $m_{l}^{(u)} \rightarrow \infty$ corresponds to the conventional Gaussian scenario, and $m_{l}^{(u)}=1 / 2$ describes the socalled one-sided Gaussian fading, i.e., the worst-case fading condition. The Rician and lognormal distributions can also be closely approximated by the Nakagami distribution in conjunction with values of $m_{l}^{(u)}>1$. The parameter $\Omega_{l}^{u}$ in (9) is the second moment of $h_{l}^{u}$, i.e., we have $\Omega_{l}^{u}=E\left[\left(\alpha_{l}^{u}\right)^{2}\right]$. We assume a negative exponentially decaying multipath intensity profile (MIP) given by $\Omega_{l}^{u}=\Omega_{1}^{u} e^{-\eta(l-1)}, \eta \geq 0$, where $\Omega_{1}^{u}$ is the average signal strength corresponding to the first resolvable path, and $\eta$ is the rate of average power decay.

We support $K$ synchronous CDMA users in the system and assume perfect power control. Consequently, when the $K$ users' signals obeying the form of (7) are transmitted over the frequency-selective fading channels characterized by (8), the received complex lowpass equivalent signal at a given mobile station can be expressed as

$$
R(t)=\sum_{k=1}^{K} \sum_{l=1}^{L} \sqrt{\frac{2 P}{U^{2}}} \mathbf{c}\left(t-\tau_{l}\right) \mathbf{B}_{U}\left(t-\tau_{l}\right) \mathbf{h}_{l}+N(t)
$$

where $N(t)$ is the complex-valued lowpass-equivalent additive white Gaussian noise (AWGN) having a double-sided spectral density of $N_{0}$, while

$$
\mathbf{h}_{l}=\left(\begin{array}{c}
h_{l}^{1} \exp \left(j \psi_{l}^{1}\right) \\
h_{l}^{2} \exp \left(j \psi_{l}^{2}\right) \\
\cdots \\
h_{l}^{U} \exp \left(j \psi_{l}^{U}\right)
\end{array}\right), \quad l=1,2, \ldots, L
$$

represents the channel's complex impulse response in the context of the $k$ th user and the $l$ th resolvable path, where $\psi_{l}^{u}=\phi_{l}^{u}-2 \pi f_{c} \tau_{l}$. Furthermore, in (10), we assumed that the signals transmitted by the $U$ number of transmission antennas arrive at the receiver antenna after experiencing the same set of delays. This assumption is justified by the fact that, in the frequency band of cellular systems, the propagation delay differences among the transmission antenna elements are on the order of nanoseconds, while the multipath delays are on the order of microseconds [2], provided that $U$ is a relatively low number.

\section{Receiver Model}

Let the first user be the user-of-interest and consider a receiver using space-time despreading as well as diversity combining, as shown in Fig. 3(b), where the subscript of the reference user's signal has been omitted for notational convenience. The receiver of Fig. 3(b) carries out the inverse processing of Fig. 3(a), in addition to multipath diversity combining. In Fig. 3(b), the received signal is first downconverted using the carrier frequency $f_{c}$, and we assumed that the receiver is capable of achieving near-perfect multipath-delay estimation for the reference user. The descrambled signal associated with the $l$ th resolvable path is space-time despread using the approach of [2], which will be further discussed in Section III, to obtain $U$ separate variables $\left\{Z_{1 l}, Z_{2 l}, \ldots, Z_{U l}\right\}$ corresponding to the $U$ parallel data bits $\left\{b_{1}, b_{2}, \ldots, b_{U}\right\}$, respectively. Following space-time despreading, a decision variable is formed for each parallel transmitted data bit of $\left\{b_{1}, b_{2}, \ldots, b_{U}\right\}$ by equal-gain (EG) diversity combining the corresponding variables associated with the $L$ number of resolvable paths, which can be written as

$$
Z_{u}=\sum_{l=1}^{L} Z_{u l}, \quad u=1,2, \ldots, U .
$$

Finally, the $U$ number of transmitted data bits $\left\{b_{1}, b_{2}, \ldots, b_{U}\right\}$ can be decided based on the decision variables $\left\{Z_{u}\right\}_{u=1}^{U}$ using the conventional decision rule of a BPSK scheme.

Above, we have described the transmitter model, the channel model, as well as the receiver model of W-CDMA using STS. Let us now describe the detection procedure of the W-CDMA scheme using STS.

\section{Detection of StS Signals}

Let $\mathbf{d}_{l}=\left[\begin{array}{llll}d_{1 l} & d_{2 l} & \cdots & d_{U l}\end{array}\right]^{T}, l=1,2, \ldots, L$-where $T$ denotes the vector transpose-represent the correlator's output 
variable vector in the context of the $l$ th $(l=1,2, \ldots, L)$ resolvable path, where

$$
d_{u l}=\int_{\tau_{l}}^{U T_{b}+\tau_{l}} R(t) c_{u}\left(t-\tau_{l}\right) d t .
$$

When substituting (10) into (13), it can be shown that

$$
\begin{gathered}
d_{u l}=\sqrt{2 P} T_{b}\left[a_{u 1} b_{u 1} h_{l}^{1} \exp \left(j \psi_{l}^{1}\right)+a_{u 2} b_{u 2} h_{l}^{2} \exp \left(j \psi_{l}^{2}\right)\right. \\
\left.+\ldots+a_{u U} b_{u U} h_{l}^{U} \exp \left(j \psi_{l}^{U}\right)\right]+J_{u}(l) \\
u=1,2, \ldots, U
\end{gathered}
$$

where

$J_{u}(l)=J_{S u}(l)+J_{M u}(l)+N_{u}(l), \quad u=1,2, \ldots, U$

and $J_{S u}(l)$ is due to the multipath-induced self-interference of the signal-of-interest inflicted upon the lth path signal, where $J_{S u}(l)$ can be expressed as

$$
\begin{aligned}
J_{S u}(l)= & \sum_{j=1, j \neq l}^{L} \sqrt{\frac{2 P}{U^{2}}} \\
& \times \int_{\tau_{l}}^{U T_{b}+\tau_{l}} \mathbf{c}\left(t-\tau_{j}\right) \mathbf{B}_{U}\left(t-\tau_{j}\right) \mathbf{h}_{j} c_{u}\left(t-\tau_{l}\right) d t .
\end{aligned}
$$

$J_{M u}(l)$ of (15) represents the multiuser interference inflicted by the signals transmitted simultaneously by the other users, which can be expressed as

$$
\begin{aligned}
J_{M u}(l)= & \sum_{k=2}^{K} \sum_{j=1}^{L} \sqrt{\frac{2 P}{U^{2}}} \\
& \times \int_{\tau_{l}}^{U T_{b}+\tau_{l}} \mathbf{c}\left(t-\tau_{j}\right) \mathbf{B}_{U}\left(t-\tau_{j}\right) \mathbf{h}_{j} c_{u}\left(t-\tau_{l}\right) d t
\end{aligned}
$$

and, finally, $N_{u}(l)$ of (15) is due to the AWGN, formulated as

$$
N_{u}(l)=\int_{\tau_{l}}^{U T_{b}+\tau_{l}} N(t) c_{u}\left(t-\tau_{l}\right) d t
$$

which is a Gaussian distributed variable having a zero mean and a variance of $2 U N_{0} T_{b}$.

Let $\mathbf{J}(l)=\left[\begin{array}{llll}J_{1}(l) & J_{2}(l) & \cdots & J_{U}(l)\end{array}\right]^{T}$. Then, the correlator's output variable vector $\mathbf{d}_{l}$ can be written as

$$
\mathbf{d}_{l}=\sqrt{2 P} T_{b} \mathbf{B}_{U} \mathbf{h}_{l}+\mathbf{J}(l), \quad l=1,2, \ldots, L
$$

where $\mathbf{B}_{U}$ is the reference user's $(U \times U)$-dimensional transmitted data matrix when we ignore the time dependence, while $\mathbf{h}_{l}$ is the channel's complex impulse response between the base station and the reference user, as shown in (11) in the context of the reference user.
Attractive STS schemes have the property [2] of $\mathbf{B}_{U} \mathbf{h}_{l}=$ $\mathbf{H}_{U} \mathbf{b}$, i.e., (19) can be written as

$$
\mathbf{d}_{l}=\sqrt{2 P} T_{b} \mathbf{H}_{U} \mathbf{b}+\mathbf{J}(l)
$$

where $\mathbf{b}=\left[\begin{array}{llll}b_{1} & b_{2} & \cdots & b_{U}\end{array}\right]^{T}$ represents the $U$ number of transmitted data bits, while $\mathbf{H}_{U}$ is a $(U \times U)$-dimensional matrix with elements from $\mathbf{h}_{l}$. Each element of $\mathbf{h}_{l}$ appears once and only once in a given row and also in a given column of the matrix $\mathbf{H}_{U}$ [2]. The matrix $\mathbf{H}_{U}$ can be expressed as

$$
\mathbf{H}_{U}(l)=\left(\begin{array}{cccc}
\alpha_{11}(l) & \alpha_{12}(l) & \cdots & \alpha_{1 U}(l) \\
\alpha_{21}(l) & \alpha_{22}(l) & \cdots & \alpha_{2 U}(l) \\
\vdots & \vdots & \ddots & \vdots \\
\alpha_{U 1}(l) & \alpha_{U 2}(l) & \cdots & \alpha_{U U}(l)
\end{array}\right)
$$

where $\alpha_{i j}(l)$ takes the form of $d_{i j} h_{l}^{m} \exp \left(j \psi_{l}^{m}\right)$, and $d_{i j} \in$ $\{+1,-1\}$ represents the sign of the $(i, j)$ th element of $\mathbf{H}_{U}$, while $h_{l}^{m} \exp \left(j \psi_{l}^{m}\right)$ belongs to the $m$ th element of $\mathbf{h}_{l}$.

It can be shown furthermore with the aid of the analysis provided in [2] that the matrix $\mathbf{H}_{U}(l)$ has the property of $\operatorname{Re}\left\{\mathbf{H}_{U}^{\dagger}(l) \mathbf{H}_{U}(l)\right\}=\mathbf{h}_{l}^{\dagger} \mathbf{h}_{l} \cdot \mathbf{I}$, where $\dagger$ denotes the complex conjugate transpose, and $\mathbf{I}$ represents a $(U \times U)$-dimensional unity matrix. Letting $\mathbf{h}_{u}(l)$ denote the $u$ th column of $\mathbf{H}_{U}(l)$, the variable $Z_{u l}$ in (12) can be formulated as [2]

$$
\begin{aligned}
Z_{u l}= & \operatorname{Re}\left\{\mathbf{h}_{u}^{\dagger}(l) \mathbf{d}_{l}\right\} \\
= & \sqrt{2 P} T_{b} b_{u} \sum_{u=1}^{U}\left|h_{l}^{u}\right|^{2}+\operatorname{Re}\left\{\mathbf{h}_{u}^{\dagger}(l) \mathbf{J}(l)\right\}, \\
& u=1,2, \ldots, U .
\end{aligned}
$$

Finally, according to (12), the decision variables associated with the $U$ parallel transmitted data bits $\left\{b_{1}, b_{2}, \ldots, b_{U}\right\}$ of the reference user can be expressed as

$$
\begin{array}{r}
Z_{u}=\sqrt{2 P} T_{b} b_{u} \sum_{l=1}^{L} \sum_{u=1}^{U}\left|h_{l}^{u}\right|^{2}+\sum_{l=1}^{L} \operatorname{Re}\left\{\mathbf{h}_{u}^{\dagger}(l) \mathbf{J}(l)\right\}, \\
u=1,2, \ldots, U
\end{array}
$$

which shows that the receiver is capable of achieving a diversity order of $U L$, as indicated by the related sums of the first term.

Above, we have analyzed the detection procedure applicable to W-CDMA signals generated using STS. Let us now derive the corresponding BER expression.

\section{BER ANALYSIS}

In this section, we derive the BER expression of the STSassisted W-CDMA system by first analyzing the statistics of the variable $Z_{u}, u=1,2, \ldots, U$, with the aid of the Gaussian approximation [14]. ${ }^{1}$ According to (23), for a given set of

\footnotetext{
${ }^{1}$ Before proceeding further, we would like to note that the validity of the Gaussian approximation has been verified by our simulations, although these results are not explicitly shown in this paper for reasons of space economy.
} 
complex channel transfer factor estimates $\left\{h_{l}^{u}\right\}, Z_{u}$ can be approximated as a Gaussian variable having a mean given by

$$
\mathrm{E}\left[Z_{u}\right]=\sqrt{2 P} T_{b} b_{u} \sum_{l=1}^{L} \sum_{u=1}^{U}\left|h_{l}^{u}\right|^{2} .
$$

Based on the assumption that the interferences imposed by the different users, by the different paths, as well as by the AWGN constitute independent random variables, the variance of $Z_{u}$ may be expressed as

$$
\begin{aligned}
\operatorname{Var}\left[Z_{u}\right] & =\mathrm{E}\left[\left(\sum_{l=1}^{L} \operatorname{Re}\left\{\mathbf{h}_{u}^{\dagger}(l) \mathbf{J}(l)\right\}\right)^{2}\right] \\
& =\sum_{l=1}^{L} \mathrm{E}\left[\left(\operatorname{Re}\left\{\mathbf{h}_{u}^{\dagger}(l) \mathbf{J}(l)\right\}\right)^{2}\right] \\
& =\frac{1}{2} \sum_{l=1}^{L} \mathrm{E}\left[\left(\mathbf{h}_{u}^{\dagger}(l) \mathbf{J}(l)\right)^{2}\right] .
\end{aligned}
$$

Substituting $\mathbf{h}_{u}(l)$, which is the $u$ th column of $\mathbf{H}_{u}(l)$ in (21), and $\mathbf{J}(l)$ having elements given by (15) into the above equation, it can be shown that for a given set of channel estimates $\left\{h_{l}^{u}\right\}$, (25) can be simplified as

$$
\begin{aligned}
\operatorname{Var}\left[Z_{u}\right] & =\frac{1}{2} \sum_{l=1}^{L} \sum_{u=1}^{U}\left|h_{l}^{u}\right|^{2} \mathrm{E}\left[\left(J_{u}(l)\right)^{2}\right] \\
& =\frac{1}{2} \sum_{l=1}^{L} \sum_{u=1}^{U}\left|h_{l}^{u}\right|^{2} \operatorname{Var}\left[J_{u}(l)\right]
\end{aligned}
$$

where $J_{u}(l)$ is given by (15). In deriving (26), we exploited the assumption of $\operatorname{Var}\left[J_{1}(l)\right]=\operatorname{Var}\left[J_{2}(l)\right]=\cdots=\operatorname{Var}\left[J_{U}(l)\right]$.

\section{A. STS-Assisted CDMA Using LS Codes}

Having characterized the various sources of interferecne, let us now demonstrate that with the advent of having an IFW, the LS codes are capable of suppressing both the MAI and MPI. More specifically, only the paths that fall outside the IFW will impose MAI and MPI on the decision. We assume having $T_{\mathrm{IFW}}=\iota T_{c}$, and the $j$ th path will inflict interference upon the $l$ th finger of the rake receiver only if we have

$$
\left|\tau_{j}-\tau_{l}\right|>\iota T_{c}
$$

which corresponds to

$$
|j-l|>\iota .
$$

Let us first consider the effect of MPI, Similarly to the benchmark of [2], it can be shown for the proposed LS code-based system that $J_{S u}(l)$ defined in (16) is also constituted by $U^{2}$ terms, and each term takes the form of

$$
\begin{array}{r}
J_{s u}(l)=\sum_{\substack{j=1 \\
|j-l|>\iota}}^{L} \sqrt{\frac{2 P}{U^{2}}} \int_{\tau_{l}}^{U T_{b}+\tau_{l}} c_{m}\left(t-\tau_{j}\right) a_{m n} b_{m n}\left(t-\tau_{j}\right) \\
\times h_{j}^{n} \exp \left(j \psi_{j}^{n}\right) c_{u}\left(t-\tau_{l}\right) d t .
\end{array}
$$

If we define the partial autocorrelation coefficient as

$$
\begin{aligned}
& \rho_{m m}(j, l)=\frac{1}{U T_{b}} \int_{0}^{\left|\tau_{j}-\tau_{l}\right|} c_{m}(t) c_{m}\left(t-\left|\tau_{j}-\tau_{l}\right|\right) d t \\
& \varrho_{m m}(j, l)=\frac{1}{U T_{b}} \int_{\left|\tau_{j}-\tau_{l}\right|}^{U T_{b}} c_{m}(t) c_{m}\left(t-\left|\tau_{j}-\tau_{l}\right|\right) d t
\end{aligned}
$$

then the integral in (29) can be expressed as

$$
\begin{aligned}
\int_{\tau_{l}}^{U T_{b}+\tau_{l}} c_{m}(t- & \left.\tau_{j}\right) c_{u}\left(t-\tau_{l}\right) b_{m n}\left(t-\tau_{j}\right) d t \\
& =\left(\rho_{m m}(j, l) b[-1]+\varrho_{m m}(j, l) b[0]\right) U T_{b} .
\end{aligned}
$$

Therefore, the corresponding MPI variance of $J_{s u}(l), u=$ $1,2, \ldots, U$ can be expressed as

$$
\begin{aligned}
\operatorname{Var}\left[J_{s u}(l)\right]=\sum_{\substack{j=1 \\
|j-l|>\iota}}^{L} & \left\{2 \Omega_{1} e^{(j-1) \eta}\left[\rho_{m m}^{2}(j, l)+\varrho_{m m}^{2}(j, l)\right]\right\} \\
& \times 2 E_{b} T_{b} \\
=\sum_{\substack{j=1 \\
|j-l|>\iota}}^{L} & \left\{2 e^{(j-1) \eta}\left[\rho_{m m}^{2}(j, l)+\varrho_{m m}^{2}(j, l)\right]\right\} \\
\times & \times 2 \Omega_{1} E_{b} T_{b} .
\end{aligned}
$$

For convenient formulation and comparison with the benchmarker STS scheme of [2], we define $\Upsilon_{S}(l)=G U \sum_{\substack{j=1 \\|j-l|>\iota}}^{L}$ $2 e^{(j-1) \eta}\left[\rho_{m m}^{2}(j, l)+\varrho_{m m}^{2}(j, l)\right]$, which is the MPI reduction factor for the $l$ th path, owing to the employment of LS codes. Then, the MPI variance of $\operatorname{Var}\left[J_{S u}(l)\right]$, which includes a total of $U^{2}$ number of $\operatorname{Var}\left[J_{s u}(l)\right]$ terms, can be approximated as

$$
\operatorname{Var}\left[J_{S u}(l)\right]_{\mathrm{II}}=\Upsilon_{S}(l) 2 \Omega_{1} E_{b} T_{b} U / G .
$$

Having characterized the MPI, let us now focus our attention on the effects of MAI. Similarly to (30) and (31), we define the partial cross-correlation coefficients as

$$
\begin{aligned}
& \rho_{u m}(j, l)=\frac{1}{U T_{b}} \int_{0}^{\left|\tau_{j}-\tau_{l}\right|} c_{u}(t) c_{m}\left(t-\left|\tau_{j}-\tau_{l}\right|\right) d t \\
& \varrho_{u m}(j, l)=\frac{1}{U T_{b}} \int_{\left|\tau_{j}-\tau_{l}\right|}^{U T_{b}} c_{u}(t) c_{m}\left(t-\left|\tau_{j}-\tau_{l}\right|\right) d t .
\end{aligned}
$$

Hence, the integral in (17) may be expressed as

$$
\begin{aligned}
\int_{\tau_{l}}^{U T_{b}+\tau_{l}} c_{m}( & \left.t-\tau_{j}\right) c_{u}\left(t-\tau_{l}\right) b_{m n}\left(t-\tau_{j}\right) d t \\
= & \left(\rho_{u m}(j, l) b_{m}[-1]+\varrho_{u m}(j, l) b_{m}[0]\right) U T_{b} .
\end{aligned}
$$


Similar to the benchmarker of [2], in the LS code-based STS scheme, the MAI term, namely $J_{M u}(l)$ defined in (17), also consists of $U^{2}$ terms, and each term takes the form of

$$
\begin{array}{r}
J_{m u}(l)=\sum_{k=2}^{K} \sum_{\substack{j=1 \\
|j-l|>\iota}}^{L} \sqrt{\frac{2 P}{U^{2}}} \int_{\tau_{l}}^{U T_{b}+\tau_{l}} c_{m}\left(t-\tau_{j}\right) a_{m n} b_{m n}\left(t-\tau_{j}\right) \\
\times h_{j}^{l} \exp \left(j \psi_{j}^{n}\right) c_{u}\left(t-\tau_{l}\right) d t
\end{array}
$$

while the variance of the MAI $J_{m u}(l)$ can be expressed as

$$
4 \Omega_{1} E_{b} T_{b} \sum_{\substack{m=1 \\ m \neq u}}^{K} \sum_{\substack{j=1 \\|j-l|>\iota}}^{L} e^{-(j-1) \eta}\left[\rho_{u m}^{2}+\varrho_{u m}^{2}\right] .
$$

Similarly to the MPI reduction factor, we define the MAI reduction factor as $\Upsilon_{M}(l)=(G U /(K-1)) \sum_{\substack{m=1 \\ m \neq u}}^{K} \sum_{\substack{j=1 \\|j-l|>>}}^{L}$ $2 e^{-(j-1) \eta}\left[\rho_{u m}^{2}+\varrho_{u m}^{2}\right]$. Then, the MAI variance $\operatorname{Var}\left[J_{M u}(l)\right]$ of the proposed LS code-based STS scheme, which includes a total of $U^{2}$ terms of the form $\operatorname{Var}\left[J_{m u}(l)\right]$, can be approximated as

$$
\operatorname{Var}\left[J_{M u}(l)\right]_{\mathrm{II}}=\Upsilon_{M}(l) \times 2(K-1) U \Omega_{1} E_{b} T_{b} U / G .
$$

\section{B. Probability of Bit Error}

Having characterized the MAI and MPI variance, let us now quantify the achievable BER performance of the proposed system. Based on analysis [2], [15], for the traditional Walshcode-based STS, the variance can be expressed as

$$
\operatorname{Var}\left[J_{u}(l)\right]=2 N_{0} U T_{b}+\frac{2 U K \Omega_{1} E_{b} T_{b}\left[q\left(L, e^{-\eta(l-1)}\right)\right]}{G} .
$$

By contrast, the corresponding variance of $J_{u}(l)$ of the LS code-based STS scheme can be expressed with the aid of (15) as

$$
\begin{aligned}
\operatorname{Var}\left[J_{u}(l)\right]=2 N_{0} U T_{b}+ & \frac{\Upsilon_{S}(l) 2 \Omega_{1} E_{b} T_{b} U}{G} \\
& +\frac{\Upsilon_{M}(l)(K-1) 2 \Omega_{1} E_{b} T_{b} U}{G} .
\end{aligned}
$$

Let us now assume that the rake receiver is capable of combining a maximum of $L_{R}$ paths' energy, owing to its complexity limitation. Then, the variance of $Z_{u}$ can be expressed as

$$
\operatorname{Var}\left[Z_{u}\right]=\frac{1}{2} \sum_{l=1}^{L_{R}} \sum_{u=1}^{U}\left|h_{l}^{u}\right|^{2} \times \operatorname{Var}\left[J_{u}(l)\right]
$$

for a given set of channel estimates $\left\{h_{l}^{u}\right\}$ using (24). Hence, the BER conditioned on $h_{l}^{u}$ for $u=1,2, \ldots, U$ and $l=$ $1,2, \ldots, L_{R}$ can be written as

$P_{b}\left(E \mid\left\{h_{l}^{u}\right\}\right)=Q\left(\sqrt{\frac{E^{2}\left[Z_{u}\right]}{\operatorname{Var}\left[Z_{u}\right]}}\right)=Q\left(\sqrt{\sum_{l=1}^{L_{R}} \sum_{u=1}^{U} 2 \gamma_{l u}}\right)$ where $Q(x)$ represents the Gaussian $Q$ function, which can also be represented in its less-conventional form as $Q(x)=$ $(1 / \pi) \int_{0}^{\pi / 2} \exp \left(-\left(x^{2} / 2 \sin ^{2} \theta\right)\right) d \theta$, where $x \geq 0$ [13], [16]. Furthermore, $2 \gamma_{l u}$ in (44) represents the output signal-tointerference-plus-noise ratio (SINR) experienced at the $l$ th finger of the rake receiver for the $u$ th STS antenna.

In both the Walsh-code-aided [2] and in the LS code-based STS scheme, $\gamma_{l u}$ of (44) is given by

$$
\gamma_{l u}=\frac{\left(\mathrm{E}\left[Z_{u l}\right]\right)^{2}}{\operatorname{Var}\left[J_{u}(l)\right]}=\bar{\gamma}_{l c} \cdot \frac{\left(h_{l}^{u}\right)^{2}}{\Omega_{1}} .
$$

However, in the Walsh-code-based STS scheme of [2], $\bar{\gamma}_{l c}$ is given by

$$
\bar{\gamma}_{l c}=\frac{1}{U}\left[\frac{K\left[q(L, \eta)-e^{-\eta(l-1)}\right]}{G}+\left(\frac{\Omega_{1} E_{b}}{N_{0}}\right)^{-1}\right]^{-1} .
$$

By contrast, in the LS code-based STS scheme, $\bar{\gamma}_{l c}$ can be expressed as

$$
\bar{\gamma}_{l c}=\frac{1}{U}\left[\frac{\Upsilon_{S}(l)}{G}+\frac{(K-1) \Upsilon_{M}(l)}{G}+\left(\frac{\Omega_{1} E_{b}}{N_{0}}\right)^{-1}\right]^{-1}
$$

where the MPI and MAI reduction factors $\Upsilon_{S}(l)$ and $\Upsilon_{M}(l)$, respectively, reflect how much interference is suppressed for the $l$ th path with the advent of the IFW, which is mainly determined by the width of the IFW and by the number of resolvable paths, i.e., by $\iota$ and $L$.

The average BER, i.e., $P_{b}(E)$, can be obtained by averaging the conditional BER expression of (44) over the joint pdf of the instantaneous SNR values corresponding to the $L_{R}$ multipath components and to the $U$ transmit antennas $\left\{\gamma_{l u}: l=\right.$ $\left.1,2, \ldots, L_{R} ; u=1,2, \ldots, U\right\}$. Since the random variables $\left\{\gamma_{l u}: l=1,2, \ldots, L_{R} ; u=1,2, \ldots, U\right\}$ are assumed to be statistically independent, the average BER can be formulated as [17]

$$
P_{b}(E)=\frac{1}{\pi} \int_{0}^{\pi / 2} \prod_{l=1}^{L_{R}} \prod_{u=1}^{U} I_{l u}\left(\bar{\gamma}_{l u}, \theta\right) d \theta
$$

where we have

$$
I_{l u}\left(\bar{\gamma}_{l u}, \theta\right)=\int_{0}^{\infty} \exp \left(-\frac{\gamma_{l u}}{\sin ^{2} \theta}\right) p_{\gamma_{l u}}\left(\gamma_{l u}\right) d \gamma_{l u} .
$$

Since both $\gamma_{l u}=\bar{\gamma}_{l c} \cdot\left(\left(h_{l}^{u}\right)^{2} / \Omega_{1}\right)$ and $h_{l}^{u}$ obey the Nakagami- $m$ distribution characterized by (9), it can be shown that the pdf of $\gamma_{l u}$ can be formulated as

$$
p_{\gamma_{l u}}\left(\gamma_{l u}\right)=\left(\frac{m_{l}}{\bar{\gamma}_{l u}}\right)^{m_{l}} \frac{\gamma^{m_{l}-1}}{\Gamma\left(m_{l}\right)} \exp \left(-\frac{m_{l} \gamma_{l u}}{\bar{\gamma}_{l u}}\right), \quad \gamma_{l u} \geq 0
$$

where $\bar{\gamma}_{l u}=\bar{\gamma}_{l c} e^{-\eta(l-1)}$ for $l=1,2, \ldots, L$. 
Upon substituting (50) into (49), it can be shown that [13]

$$
I_{l u}\left(\bar{\gamma}_{l u}, \theta\right)=\left(\frac{m_{l}^{u} \sin ^{2} \theta}{\bar{\gamma}_{l u}+m_{l}^{u} \sin ^{2} \theta}\right)^{m_{l}^{u}} .
$$

Finally, upon substituting (51) into (48), the average BER of the STS-assisted W-CDMA system using $U$ transmission antennas can be written as

$$
P_{b}(E)=\frac{1}{\pi} \int_{0}^{\pi / 2} \prod_{l=1}^{L_{R}} \prod_{u=1}^{U}\left(\frac{m_{l}^{u} \sin ^{2} \theta}{\bar{\gamma}_{l u}+m_{l}^{u} \sin ^{2} \theta}\right)^{m_{l}^{u}} d \theta
$$

which shows that the diversity order achieved is $L_{R} U$, namely the product of the diversity due to STS and the diversity contributed by the rake receiver. Furthermore, if we assume that $m_{l}$ is independent of $u$, i.e., that all of the parallel transmitted signals experience an identical Nakagami fading, then (52) can be expressed as

$$
P_{b}(E)=\frac{1}{\pi} \int_{0}^{\pi / 2} \prod_{l=1}^{L_{R}}\left(\frac{m_{l} \sin ^{2} \theta}{\bar{\gamma}_{l u}+m_{l} \sin ^{2} \theta}\right)^{U m_{l}} d \theta .
$$

\section{Numerical Results}

Having characterized the analytical performance of the system, let us now consider the achievable BER performance. Stańczak et al. [4] concluded that when using LS codes, the width $\iota$ of the IFW and the number of users $K$ has to obey the following:

$$
(\iota-1) K \leq G .
$$

Furthermore, LS codes require $W_{0}$ number of zero-valued chips, which are inserted in the beginning and center of the code sequence for creating the IFW. In our scenario, the $\mathrm{LS}\left(N, P, W_{0}\right)=\mathrm{LS}(4,32,4)$ codes having a length of $L_{S}=$ $N P+2 W_{0}=136$ were invoked, and their effective spreading gain was $L_{S}=N P=128$ since the zero-valued chips do not include the spreading gain. For the sake of maintaining the same chip rate and same spectral efficiency for both STS schemes, we set the spreading gain of the traditional STS-assisted CDMA system to $G^{\prime}=N P+2 W_{0}=136$. Furthermore, for the sake of simplicity, we assume that all paths have the same Nakagami fading parameter, i.e., $m_{l}=m$ and $l=0, \ldots, L_{r}-1$.

We assume that the chip rate is $1.2288 \mathrm{M}$ chip/s, the channel's delay spread is negatively exponentially distributed having a uniformly distributed mean delay in the range of $[0.3,3] \mu \mathrm{s}$ [18], and we assume that both the random and large-area synchronized (LAS) code-based systems have a chip rate of $1.2288 \mathrm{M}$ chips. The number of resolvable paths is $L_{p}=\left\lfloor\tau / T_{c}\right\rfloor+1=4$, where we have $\tau=3 \mu \mathrm{s}$. Both the traditional STS and the LS code-based STS schemes supported $K=32$ users, and the width of the IFW of the LS codes was $\iota=3$. We can see from Fig. 4 that the LS code-based STS scheme exhibits a significantly better performance than the traditional Walsh-code-based system having the same diversity order of $L_{R} U$. The reason that the LS code-based STS scheme

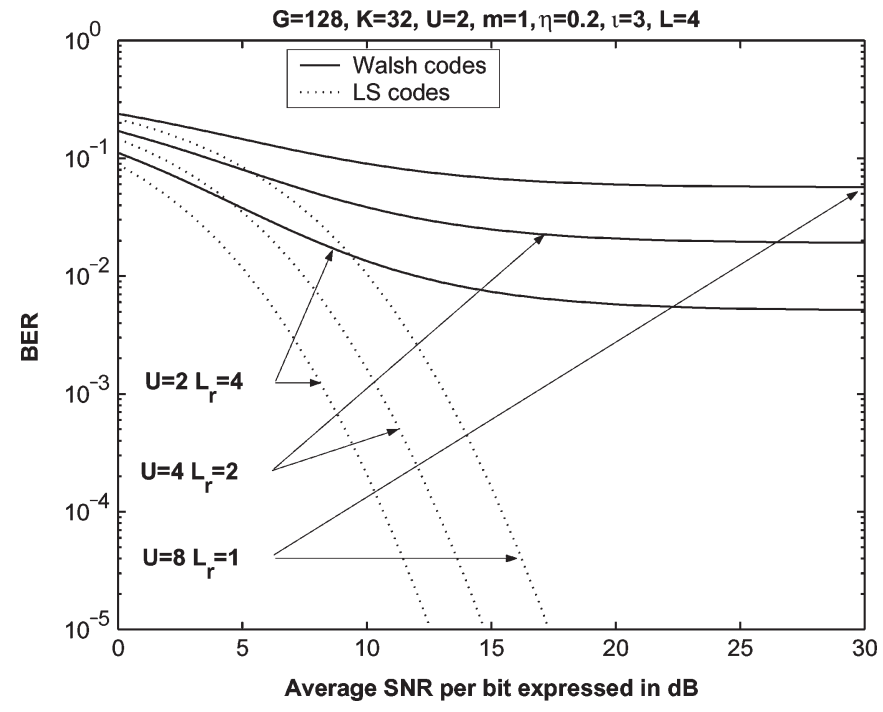

Fig. 4. BER versus SNR per bit $\left(E_{b} / N_{0}\right)$ and performance comparison between the Walsh-code-based and LS code-based STS transmit diversity schemes having the same diversity order of $L_{R} \cdot U$ when communicating over a Nakagami- $m(m=1)$ fading multipath $(L=4)$ channel evaluated from (53) by assuming that the average power decay rate was $\eta=0.2$. The remaining system parameters are listed at the top of the figure.

outperforms the traditional STS scheme is that the MAI and MPI are reduced as a benefit of using LAS codes, which was quantified by (53). Fig. 5 characterizes the achievable performance of these two schemes communicating over different fading channels associated with different Nakagami fading parameters. More explicitly, when we have $m=1$, we model a Rayleigh fading channel, $m=2$ represents a Rician fading channel, while $m \rightarrow \infty$ corresponds to an AWGN channel. From this figure, we can observe that the LS code-based STS scheme exhibited a better performance than the traditional STS scheme regardless of the value of $m$. More specifically, provided that we have $L_{p}=4$, the LS code-based STS scheme outperformed the traditional STS scheme when communicating over different Nakagami multipath fading channels.

Fig. 6 shows the performance of these two systems for transmission over different dispersive channels having $L=4, \ldots, 12$ resolvable multipath components but assuming that only $L_{r}=3$ of these components were combined by the rake receiver owing to its limited affordable complexity. From Fig. 6, we may conclude that the LS codes are effective when the number of resolvable paths is relatively low, for example, when we have $L=4$. When $L$ is increased to 8 , the LS codebased STS scheme only has a slight gain over the traditional STS scheme, while when $L$ is increased to 12 , the LS codebased STS scheme performs slightly worse than the traditional STS scheme. The reason for this performance erosion is that many of the paths will be located outside the IFW when $L_{p}$ is high, and both the autocorrelation and cross correlation of the LS codes outside the IFW are higher than those of the random codes. Hence, many of the multipath components arrive outside the IFW when $L$ is high, which inevitably will increase both the MAI and MPI. However, the same LS codes may be reused in the interfering cells since their autocorrelation is also zero within the IFW. As far as the employment of the cell-specific scrambling codes of the $3 \mathrm{G}$ systems is concerned, their role 


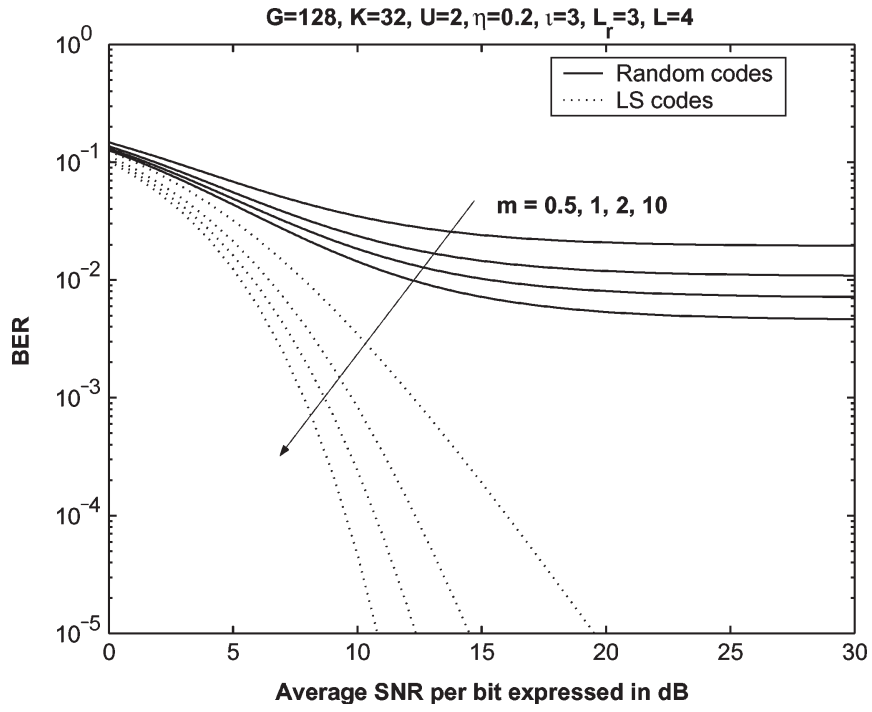

Fig. 5. BER versus SNR per bit $\left(E_{b} / N_{0}\right)$ and performance comparison between the Walsh-code-based and LS code-based STS transmit diversity schemes when communicating over various Nakagami- $m$ fading multipath ( $L=4)$ channels, where $L_{r}=3$ out of the $L=3$ available paths were combined by the rake receiver. The remaining system parameters are listed at the top of the figure.

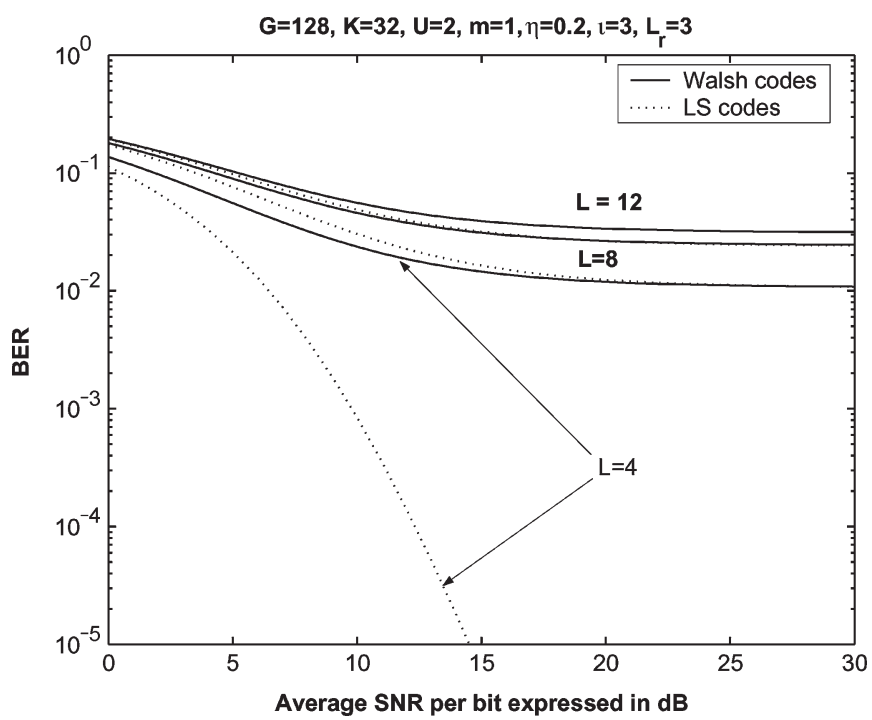

Fig. 6. BER versus SNR per bit $\left(E_{b} / N_{0}\right)$ and performance comparison between the Walsh-code-based and LS code-based transmit diversity schemes and the conventional rake receiver arrangement when communicating over different dispersive Nakagami- $m$ channels having $L=4,8$, and 12 resolvable paths but only combining $L_{R}=3$ of them, owing to the maximum complexity limitations.

is replaced by using cell-specific LA codes in the LAS 2000 CDMA system proposal [19], which allows the system to retain an IFW.

To circumvent the performance limitation of the proposed system in case of high delay spreads, we finally introduce the concept of MC LAS DS-CDMA, which allows us to extend the IFW duration by a factor corresponding to the number of subcarriers. Fig. 7 demonstrates the achievable performance of single-carrier (SC) LS code-assisted STS and MC LS codebased STS for a $3.884 \mathrm{M}$ chips/s system. From this figure, we may conclude that the LS code-based STS-assisted MC

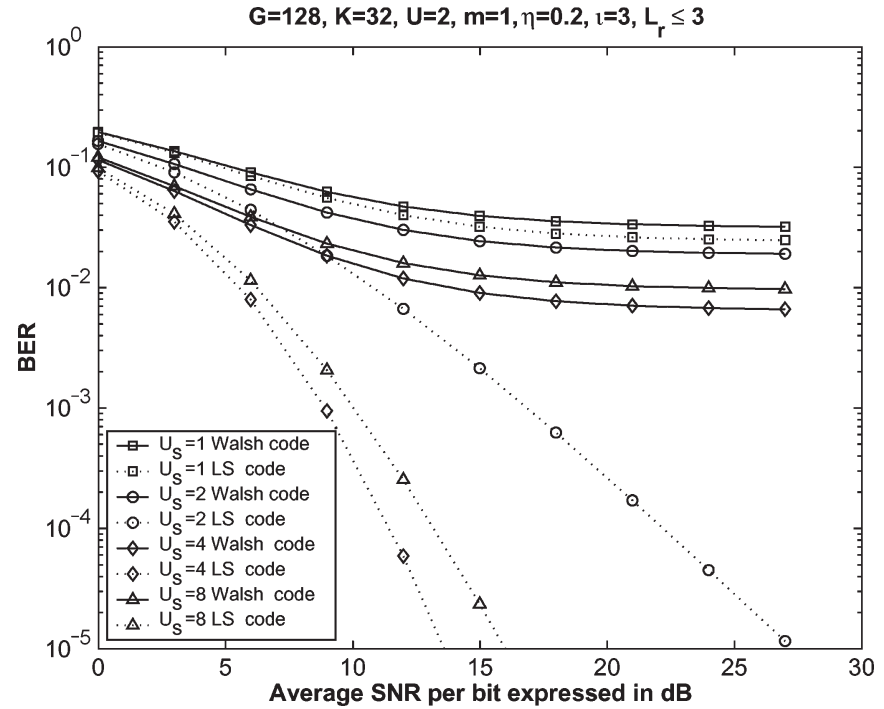

Fig. 7. BER versus SNR per bit $\left(E_{b} / N_{0}\right)$ and performance comparison between the Walsh-code-based and LS code-based transmit diversity schemes and the conventional rake receiver arrangement when invoking MC CDMA, where the number of subcarriers $U_{s}$ is 1,2, 4, and 8, respectively. Furthermore, only $L_{R}=3$ path have been combined, owing to the maximum complexity limitations imposed.

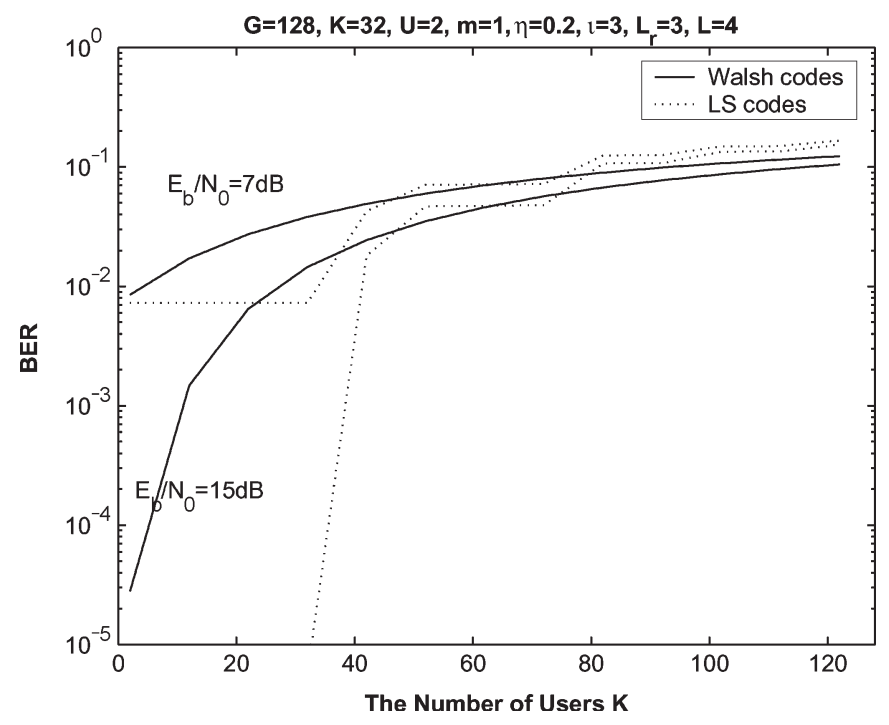

Fig. 8. BER versus SNR per bit $\left(E_{b} / N_{0}\right)$ and performance comparison between the Walsh-code-based and LS code-based STS transmit diversity schemes as a function of the number of users $K$. The remaining system parameters are listed at the top of the figure.

DS-CDMA scheme is capable of achieving the best performance tradeoff by selecting the optimum number of subcarriers $U_{s}$ according to the channel's delay dispersion $\tau_{\mathrm{ch}}$. For example, we may conclude from Fig. 7 that the MC LAS DS-CDMA system using $U_{s}=4$ subcarriers exhibited the best tradeoffs in a scenario having a delay spread of $\tau_{\mathrm{ch}}=3 \mu \mathrm{s}$.

From Fig. 8, we can observe that if the system's user load is high, the LS code-based STS scheme will have no advantage over the traditional Walsh-code-based STS scheme of [2], which is caused by two factors. First, the number of LS codes having an IFW of $\iota=3$ is limited. For example, when we consider $G=128$, only 32 LS codes have an IFW of $\iota=3$, and when the number of users $K$ exceeds 32 , the width of 
the IFW will be reduced to zero since even the codes having $\iota=0$ will be required for supporting $K \geq 32$ users. In this scenario, the LS code-based STS scheme becomes incapable of effectively suppressing the MAI and MPI. Second, it may be shown that the cross correlation of LS codes outside the IFW is higher than that of the random codes; hence, LS codes may impose slightly increased interferences when the number of users $K$ is increased. Therefore, the LS code-based STS scheme is more effective in low-user-load scenarios, i.e., when we have $K \leq G / 3$.

\section{CONCLUSiON}

The proposed LS code-based STS scheme exhibited a significantly better performance than that of the traditional Walshcode-based STS scheme [2] when the number of users supported does not exceed $G / 3$. As the number of resolvable paths $L$ of the channel increases, the LS code-based STS scheme only has a slight gain over the traditional STS scheme [2], owing to the fact that many of the paths arrive outside the IFW and because the autocorrelation and cross correlation of LS codes outside the IFW are higher than those of the random codes. Furthermore, when communicating in a high-user-load scenario, for example, when we have $K=G$, the LS codebased STS scheme may exhibit a worse performance than the traditional STS scheme of [2].

\section{REFERENCES}

[1] L. Hanzo, T. H. Liew, and B. L. Yeap, Turbo Coding, Turbo Equalisation and Space-Time Coding for Transmission Over Fading Channels. Piscataway, NJ: IEEE Press, 2002.

[2] B. Hochwald, T. L. Marzetta, and C. B. Papadias, "A transmitter diversity scheme for wideband CDMA systems based on space-time spreading," IEEE J. Sel. Areas Commun., vol. 19, no. 1, pp. 48-60, Jan. 2001.

[3] L. Hanzo, L. L. Yang, E. L. Kuan, and K. Yen, Single- and Multi-Carrier DS-CDMA. Piscataway, NJ: IEEE Press, 2003. 1060 pages.

[4] S. Stańczak, H. Boche, and M. Haardt, "Are LAS-codes a miracle?" in Proc. GLOBECOM, San Antonio, TX, Nov. 2001, vol. 1, pp. 589-593.

[5] B. J. Choi and L. Hanzo, "On the design of LAS spreading codes," in Proc. IEEE VTC Conf.-Fall, Vancouver, BC, Canada, Sep. 2002, pp. $2172-2176$.

[6] C.-C. Tseng and C. L. Liu, "Complementary sets of sequences," IEEE Trans. Inf. Theory, vol. IT-18, no. 5, pp. 644-652, Sep. 1972.

[7] R. L. Frank, "Polyphase complementary codes," IEEE Trans. Inf. Theory, vol. IT-26, no. 6, pp. 641-647, Nov. 1980.

[8] R. Sivaswamy, "Multiphase complementary codes," IEEE Trans. Inf. Theory, vol. IT-24, no. 5, pp. 546-552, Sep. 1978.

[9] J. G. Proakis, Digital Communications, 3rd ed. New York: McGrawHill, 1995.

[10] N. Nakagami, "The $m$-distribution, a general formula for intensity distribution of rapid fading," in Statistical Methods in Radio Wave Propagation, W. G. Hoffman, Ed. Oxford, U.K.: Pergamon, 1960.

[11] T. Eng and L. B. Milstein, "Coherent DS-CDMA performance in Nakagami multipath fading," IEEE Trans. Commun., vol. 43, no. 2-4, pp. 1134-1143, Feb.-Apr. 1995.

[12] V. Aalo, O. Ugweje, and R. Sudhakar, "Performance analysis of a DS/CDMA system with noncoherent $M$-ary orthogonal modulation in Nakagami fading," IEEE Trans. Veh. Technol., vol. 47, no. 1, pp. 20-29, Feb. 1998

[13] M.-S. Alouini and A. J. Goldsmith, "A unified approach for calculating error rates of linearly modulated signals over generalized fading channels," IEEE Trans. Commun., vol. 47, no. 9, pp. 1324-1334, Sep. 1999.

[14] M. B. Pursley, "Performance evaluation for phase-coded spread-spectrum multiple-access communication-Part I: System analysis," IEEE Trans. Commun., vol. COM-25, no. 8, pp. 795-799, Aug. 1977.

[15] L.-L. Yang and L. Hanzo, "Performance of broadband multicarrier DSCDMA using space-time spreading assisted transmit diversity," IEEE Trans. Wireless Commun., vol. 4, no. 3, pp. 885-894, May 2005.
[16] M. K. Simon and M.-S. Alouini, "A unified approach to the probability of error for noncoherent and differentially coherent modulation over generalized fading channels," IEEE Trans. Commun., vol. 46, no. 12, pp. 1625-1638, Dec. 1998.

[17] _ "A unified approach to the performance analysis of digital communication over generalized fading channels," Proc. IEEE, vol. 86, no. 9, pp. 1860-1877, Sep. 1998.

[18] W. C. Y. Lee, Mobile Communications Engineering, 2nd ed. New York: McGraw-Hill, 1998.

[19] Physical Layer Aspects of TD-LAS High Speed Packet Technology, Jul. 2001. CWTS-SWG2, LAS-CDMA.

Hua Wei received the B.Eng. degree in wireless communication from Beijing University of Posts and Telecommunication, Beijing, China, in 1997 and the $\mathrm{Ph} . \mathrm{D}$. degree from the School of Electronics and Computer Science, University of Southampton, Southampton, U.K., in 2005.

He was with Hua Wei Technology Company, working on synchronous digita hierarchy system software development and with Motorola China Software Center, working on code-division multiple-access system (CDMA) software development. Since 2001, he has been with the Communications Research Group, School of Electronics and Computer Science, University of Southampton, Southampton, U.K., and has been involved in researching CDMA schemes designed for future generations of wireless mobile communication systems. He is the author or coauthor of about 30 papers.

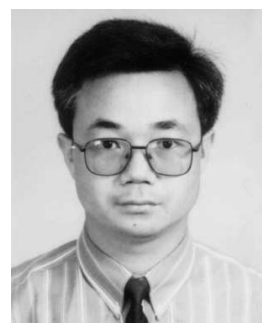

Lie-Liang Yang (M'98-SM'02) received the B.Eng. degree in communications engineering from Shanghai TieDao University, Shanghai, China, in 1988 and the M.Eng. and Ph.D. degrees in communications and electronics from Northern Jiaotong University, Beijing, China, in 1991 and 1997, respectively.

Since December 1997, he has been with the Communications Research Group, School of Electronics and Computer Science, University of Southampton, Southampton, U.K., where he held various research posts as a Visiting Postdoctoral Research Fellow, a Research Fellow, and a Senior Research Fellow. He currently holds an academic post as a Reader. From June 1997 to December 1997, he was a Visiting Scientist with the Institute of Radio Engineering and Electronics, Academy of Sciences of the Czech Republic, Prague. He has been involved in a number of projects funded by the National Sciences Foundations of China, the Grant Agency of the Czech Republic, the Engineering and Physical Sciences Research Council of the U.K., and the European Union. He is the author or coauthor of more than 100 papers published in various journals and conference proceedings. His research has covered a wide range of areas in communications, which include data networks and security, intelligent wireless networking, error control coding, modulation and demodulation, spread-spectrum communications and multiuser detection, pseudonoise code synchronization, smart antennas, adaptive wireless systems, as well as wideband, broadband, and ultrawideband code-division multiple access for advanced wireless mobile communication systems.

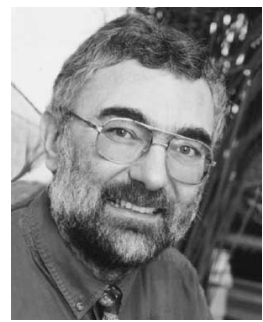

Lajos Hanzo (M'91-SM'92-F'04) received the Master's degree (Dipl. Ing.) in electronics and the $\mathrm{Ph} . \mathrm{D}$. degree from the Technical University of $\mathrm{Bu}-$ dapest, Budapest, Hungary, in 1976 and 1983, respectively, and the D.Sc. degree from the University of Southampton, Southampton, U.K., in 2004.

During his 30-year career in telecommunications, he has held various research and academic posts in Hungary, Germany, and the U.K. Since 1986, he has been with the School of Electronics and Computer Science, University of Southampton, where he holds the Chair in telecommunications. Currently, he is managing an academic research team, working on a range of research projects in the field of wireless multimedia communications sponsored by industry, the Engineering and Physical Sciences Research Council of U.K., the European IST Programme, and the Mobile Virtual Centre of Excellence (VCE) of the U.K. He is an enthusiastic supporter of industrial and academic liaison, and he offers a range of industrial courses. He has coauthored 12 John Wiley/IEEE Press books totaling about 9000 pages on mobile radio communications, published more than 600 research papers, organized and chaired conference sessions, presented overview lectures, and been awarded a number of distinctions.

Dr. Hanzo is a Fellow of the Royal Academy of Engineering and the Institution of Electrical Engineers. He is an IEEE Distinguished Lecturer of both the Communications Society and the Vehicular Technology Society. He is a Governor of the IEEE Vehicular Technology Society and an Executive Board Member of the Pan-European Newcom consortium. 